\title{
Performance of Linear Receivers in Frequency-Selective MIMO Channels
}

\author{
Ahmed Hesham Mehana, Student Member, IEEE, and Aria Nosratinia, Fellow, IEEE
}

\begin{abstract}
This paper investigates the performance of the zero-forcing (ZF) and minimum mean-square error (MMSE) equalizers in MIMO frequency selective channels under zeropadding (ZP) transmission. It was previously known that the SISO zero-forcing ZP receiver achieves full diversity; this paper shows that the MIMO version of this receiver is suboptimal in diversity. The MMSE $\mathrm{ZP}$ is also investigated, showing that it exhibits an intricate error behavior that depends not only on channel memory and antenna configuration, but also on transmission rate. This behavior is fully characterized in closed form, revealing that the MMSE ZP receiver attains the (optimal) diversity of a ML receiver but only at small spectral efficiencies. Thus MMSE ZP works better than ZF ZP, but not quite as well as ML. To further improve the performance, lattice-reduction aided equalization in the frequency-selective channel is investigated. It is shown that lattice-reduction-aided zero forcing equalizer as well as MMSE equalizer achieve the maximum spatial and temporal diversity at all spectral efficiencies.
\end{abstract}

Index Terms-MIMO, MMSE, ZF, diversity, lattice-reduction.

\section{INTRODUCTION}

I $\mathrm{N}$ block transmission schemes a guard interval is inserted between consecutive blocks to avoid inter-block interference. This guard interval can either consist of zero symbols, known as zero padding (ZP), or it can consist of a cyclic extension of the transmitted symbols, known as cyclic prefix (CP). It is known that in single-antenna (SISO) systems ZP transmission allows a linear receiver (e.g. zero-forcing and MMSE) to collect the full multipath diversity [1], [2]. The corresponding question for MIMO systems has been open up to date, which is the subject of the first part of this paper.

We discover that in MIMO systems, zero forcing with zero padding does not achieve full diversity except for the special case of SISO and SIMO systems. The MMSE receiver provides better performance in a manner depending not only on antenna and channel configuration, but also on transmission rate. The MMSE receiver attains optimal diversity for a small set of rates. In search for better performance, we consider lattice reduction (LR), a technique that has been proposed to reduce the gap between the performance of linear receivers and maximum likelihood receivers [3], [4]. This technique has been used mostly on single-user and multi-user flat MIMO

Manuscript received May 10, 2012; revised November 1, 2012 and March 1, 2013, accepted March 2, 2013.

The authors are with the Department of Electrical Engineering, The University of Texas at Dallas, Richardson, TX 75083-0688 USA (e-mail: \{ahmed.mehana, aria\}@utdallas.edu).

The work in this paper was presented in part at the IEEE International Symposium on Information Theory (ISIT), 2011.

Digital Object Identifier 10.1109/TCOMM.2013.050313.120666 channels or frequency-selective SISO channel [5]-[8]; in this work we apply it to the frequency-selective MIMO channel. We adopt the complex Lenstra-Lenstra-Lovász (CLLL) algorithm [8] and analytically obtain the diversity order of LRaided ZF and MMSE over frequency selective MIMO.

We now briefly review some related results in the area of linear receivers. Onggosanusi et al. [9] study the outage probability of different finite-length equalizers in scalar and vector frequency-selective channels. Hedayat and Nosratinia [10] obtain the diversity order of block-based linear equalizers (LE) under CP transmission. They show that the diversityorder of ZF-LE is always one whereas the diversity-order of MMSE-LE depends on the spectral efficiency $R(\mathrm{~b} / \mathrm{s} / \mathrm{Hz})$ via a relationship that was subsequently given explicitly by [11]. For the infinite-length equalizers, the diversity order was obtained in [12] for ZF-LE and MMSE-LE. Slock [13] obtains the DMT of IIR equalizers in time-varying frequency-selective channel under CP transmission as well as the DMT of FIR equalizers in block transmission (but without $\mathrm{ZP} / \mathrm{CP}$ insertion). The performance of the MMSE receivers in the context of MIMO channels was also studied in [9], [14], [15]. The diversity of the MMSE MIMO receivers at zero multiplexing gain $^{1}$ was shown to be a function of the spectral efficiency $R$ [14], [15]. For the frequency-selective channel, Medles et al. [16] characterize the optimal DMT of the i.i.d. Rayleigh MIMO channel. Slock [17] analyzes the diversity of linear receivers in frequency selective channel under cyclic-prefix transmission.

To reduce the gap between the performances of linear receivers and the maximum likelihood receiver, the lattice reduction technique has been recently proposed. Lattice reduction [3], [4] aims to find a better representation of the channel matrix that has nearly orthogonal columns vectors, thus the noise enhancement problem inherent in channel inversion is reduced. It has been shown through simulations [3], [4], [18] (and analytical results for some cases [5]-[8]) that the diversity order of LR-aided receiver is the same as maximum likelihood. In this paper we analytically obtain the diversity order of LRaided ZF over frequency selective MIMO channels.

This paper is organized as follows. Section II describes the system model. Section III finds the exponential order of zero-forcing receiver under-zero-padding receiver. Section V provides the analysis of lattice-reduction aided equalization. Section VI provides simulation results.

\footnotetext{
${ }^{1}$ Recall that zero multiplexing gain means that the spectral efficiency $R$ is not a function of the signal-to-noise-ratio.
} 


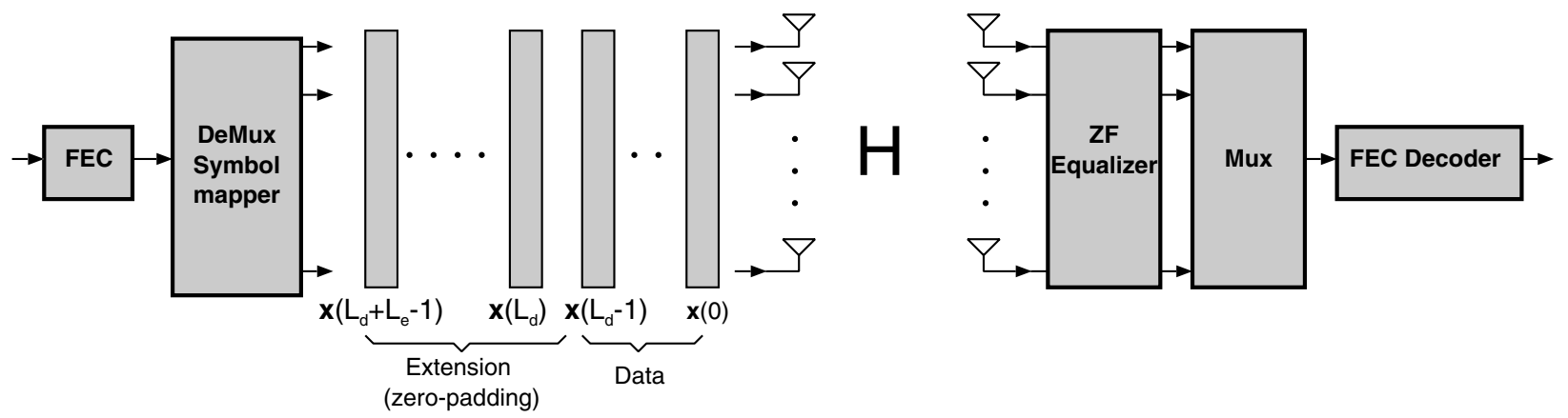

Fig. 1. Single-carrier block transmission in a frequency selective channel.

\section{SySTEM Model}

Figure 1 shows a MIMO transmission model. We assume a frequency-selective channel with channel memory $\nu$. The $(\nu+1)$-tap channel impulse response between the transmit antenna $m$ and receive antenna $n$ is given by $\mathbf{h}_{m n}=$ $\left[h_{m n, 0}, h_{m n, 1}, \ldots, h_{m n, \nu}\right]$. We also assume that $\mathbf{h}_{m n}$ remains unchanged during a transmission block. Assuming $M$ transmit and $N$ receive antennas with $N \geqslant M$, the received vector $\mathbf{y}_{k}$ at time instant $k$ is given by [11], [19]

$$
\mathbf{y}_{k}=\sum_{i=0}^{\nu} \mathbf{H}_{i} \mathbf{x}_{k-i}+\mathbf{n}_{k}
$$

where $\mathbf{H}_{i}$ is the $N \times M$ channel matrix that has $h_{m n, i}$ as its $(m, n)$ element, $\mathbf{x}_{k-i}$ is $M \times 1$ transmitted vector at time index $k-i$, and $\mathbf{n}_{k}$ is the $N \times 1$ Gaussian noise vector at time index $k$. The number of transmitted $M \times 1$ data blocks is $L_{d}$ and the length of zero-blocks is $L_{e}$ (cf. Figure 1). The total number of transmission times in the block is denoted $L \triangleq L_{d}+L_{e}$. Stacking the transmitted vectors in an $M L \times 1$ vector, we can rewrite the model in (1)

$$
\overline{\mathbf{y}}=\mathbf{H}_{\mathrm{e}} \overline{\mathbf{x}}+\overline{\mathbf{n}}
$$

where $\overline{\mathbf{y}}$ is the $L_{d} N \times 1$ received vector, $\overline{\mathbf{x}}$ is the $M L \times 1$ transmitted vector, $\overline{\mathbf{n}}$ is the $N L_{d} \times 1$ white Gaussian noise vector and $\mathbf{H}_{\mathbf{e}}$ is the $N L \times M L_{d}$ channel matrix given by

$$
\mathbf{H}_{\mathbf{e}}=\left[\begin{array}{cccc}
\mathbf{H}_{0} & \mathbf{0} & \cdots & \mathbf{0} \\
\mathbf{H}_{1} & \mathbf{H}_{0} & \ddots & \vdots \\
\vdots & \mathbf{H}_{1} & \ddots & \vdots \\
\mathbf{H}_{\nu} & \vdots & \ddots & \mathbf{H}_{0} \\
\mathbf{0} & \mathbf{H}_{\nu} & \ddots & \mathbf{H}_{1} \\
\vdots & \mathbf{0} & \ddots & \vdots \\
\vdots & \vdots & \cdots & \mathbf{H}_{\nu}
\end{array}\right]
$$

The MMSE/ZF equalizer and the unbiased decision-point SINR of its output are respectively given by [20]:

$$
\begin{aligned}
\mathbf{W} & =\left(\mathbf{H}_{\mathbf{e}}{ }^{H} \mathbf{H}_{\mathbf{e}}+c \rho^{-1} \mathbf{I}\right)^{-1} \mathbf{H}_{\mathbf{e}}{ }^{H} \\
\gamma_{k} & =\frac{1}{\left[\left(c \mathbf{I}+\rho \mathbf{H}_{\mathbf{e}}{ }^{H} \mathbf{H}_{\mathbf{e}}\right)^{-1}\right]_{k k}}-c \quad k=1, \ldots, M L_{d}
\end{aligned}
$$

where $\rho$ is the per-stream SNR. The constant $c=0$ for the ZF equalizer and $c=1$ for the MMSE equalizer. The diversity gain is given by

$$
d \triangleq-\lim _{\rho \rightarrow \infty} \frac{\log P(E)}{\log \rho}
$$

where $P(E)$ is the pairwise error probability. Following the methodology of [11], [21], we perform the outage analysis to obtain $d_{\text {out }}$ defined as

$$
d_{\text {out }} \triangleq-\lim _{\rho \rightarrow \infty} \frac{\log P_{\text {out }}}{\log \rho}
$$

where $P_{\text {out }}$ is the outage probability. Providing tight exponential bounds on the error probability via outage (hence $d=d_{\text {out }}$ ) follows from [11], and omitted here for brevity. We also denote the exponential equality of two functions $f(\rho)$ and $g(\rho)$ as $f(p) \doteq g(p)$ when

$$
\lim _{\rho \rightarrow \infty} \frac{\log f(\rho)}{\log (\rho)}=\lim _{\rho \rightarrow \infty} \frac{\log g(\rho)}{\log (\rho)}
$$

Similarly to [21], in our analysis we substitute $R=r \log \rho$ where $r$ is the multiplexing gain. When $r=0, R$ is not a function of $\rho$ and in this case the diversity must be obtained via a different type of analysis [15], [22]. In the following sections we analyze the outage diversity for the zero padding systems. The PEP analysis follows in a direct manner as in the flat fading case [15].

\section{The Zero Padding ZF Receiver}

The equalized signal is given by

$$
\hat{\mathbf{y}} \triangleq \mathbf{W} \overline{\mathbf{y}}=\mathbf{W} \overline{\mathbf{x}}+\tilde{\mathbf{n}}
$$

where $\tilde{\mathbf{n}}=\mathbf{W} \overline{\mathbf{n}}$. Assuming joint spatial encoding, the effective mutual information between the data vector $\hat{\mathbf{x}}$ and the equalized received signal $\hat{\mathbf{y}}$ is ${ }^{2}$ [9], [11]

$$
I(\hat{\mathbf{x}}, \hat{\mathbf{y}})=\sum_{k=1}^{M L_{d}} I\left(\hat{x}_{k}, \hat{y}_{k}\right)=\sum_{k=1}^{M L_{d}} \log \left(1+\gamma_{k}\right) .
$$

It is well known that linear receivers can achieve full multipath diversity under zero-padding transmission in SISO frequency-selective channel [1], [23], [24]. In this section we investigate the corresponding MIMO channel.

\footnotetext{
${ }^{2}$ Because the resulting streams will be processed independently by the ensuing decoders. In other words, any residual interference beyond the equalizer is treated as noise.
} 
1) Diversity Upper Bound: Assuming $R$ is the normalized rate in bits $/ \mathrm{s} / \mathrm{Hz}$, the outage can be expressed:

$$
P_{\text {out }} \triangleq \mathbb{P}\left(\frac{1}{L} I(\hat{\mathbf{x}} ; \hat{\mathbf{y}})<R\right) \text {. }
$$

Applying the ZF equalizer to the received vector in (2) the outage probability is given by

$$
\begin{aligned}
P_{\text {out }} & =\mathbb{P}\left(\frac{1}{L} \sum_{k=1}^{M L_{d}} \log \left(1+\gamma_{k}\right)<R\right) \\
& =\mathbb{P}\left(\frac{1}{L} \sum_{k=1}^{M L_{d}} \log \left(1+\frac{\rho}{\left[\left(\mathbf{H}_{\mathbf{e}}{ }^{H} \mathbf{H}_{\mathbf{e}}\right)^{-1}\right]_{k k}}\right)<R\right) \\
& \doteq \mathbb{P}\left(\frac{1}{L} \sum_{k=1}^{M L_{d}} \log \frac{\rho}{\left[\left(\mathbf{H}_{\mathbf{e}}{ }^{H} \mathbf{H}_{\mathbf{e}}\right)^{-1}\right]_{k k}}<R\right) \\
& \geqslant \mathbb{P}\left(\frac{L_{d} M}{L} \log \frac{1}{M L_{d}} \sum_{k=1}^{M L_{d}} \rho^{-1}\left[\left(\mathbf{H}_{\mathbf{e}}{ }^{H} \mathbf{H}_{\mathbf{e}}\right)^{-1}\right]_{k k}>-R\right) \\
& =\mathbb{P}\left(\sum_{k=1}^{M L_{d}} \frac{1}{\rho \lambda_{k}\left(\mathbf{H}_{\mathbf{e}}{ }^{H} \mathbf{H}_{\mathbf{e}}\right)}>M L_{d} 2^{-\frac{R}{M \alpha}}\right)
\end{aligned}
$$

where we substitute (4) in (7) to obtain (8), and (10) follows in the same manner as the bound in [25, Section III.B]. In (11) we have introduced a new parameter $\alpha \triangleq \frac{L_{d}}{L}$. The $\lambda_{k}(\mathbf{G})$ denote the $k$-th eigenvalue of a square matrix $\mathbf{G}$.

The block diagonal elements of $\mathbf{H}_{\mathbf{e}}{ }^{H} \mathbf{H}_{\mathbf{e}}$ are given by

$$
\mathbf{D}=\sum_{i=0}^{\nu} \mathbf{H}_{i}^{H} \mathbf{H}_{i}
$$

The matrix $\mathbf{H}_{\mathbf{e}}{ }^{H} \mathbf{H}_{\mathbf{e}}$ is Toeplitz and Hermitian. Note that $\mathbf{D}$ is a Wishart matrix. ${ }^{3}$ Define $M_{S}$ to be the set of the $M$ smallest eigenvalues of $\mathbf{H}_{\mathbf{e}}{ }^{H} \mathbf{H}_{\mathbf{e}}$. The outage bound in (11) can be further lower bounded as follows

$$
P_{\text {out }} \geqslant \mathbb{P}\left(\sum_{k: \lambda_{k} \in M_{S}} \frac{1}{\rho \lambda_{k}\left(\mathbf{H}_{\mathbf{e}}{ }^{H} \mathbf{H}_{\mathbf{e}}\right)}>M L_{d} 2^{-\frac{R}{M \alpha}}\right) .
$$

Note that the sum in (13) is over a smaller set of $M$ positive elements. We now use the Sturmian separation theorem [26, P.1077].

Theorem 1: (Sturmian Separation Theorem) Let $\left\{\mathbf{A}_{r}, r=\right.$ $1,2, \ldots\}$ be a sequence of symmetric $r \times r$ matrices such that each $\mathbf{A}_{r}$ is a submatrix of $\mathbf{A}_{r+1}$. Then if $\left\{\lambda_{k}\left(\mathbf{A}_{r}\right), k=\right.$ $1, \ldots, r\}$ denote the ordered eigenvalues of each matrix $\mathbf{A}_{r}$ in descending order, we have

$$
\lambda_{k+1}\left(\mathbf{A}_{i+1}\right) \leq \lambda_{k}\left(\mathbf{A}_{i}\right) \leq \lambda_{k}\left(\mathbf{A}_{i+1}\right) .
$$

For our purposes, we consider a special case of the Sturmian Theorem by constructing a set of matrices $\mathbf{A}_{M}, \mathbf{A}_{M+1}, \ldots, \mathbf{A}_{L_{d} M}$ starting by the largest one $\mathbf{A}_{L_{d} M} \triangleq$ $\mathbf{H}_{\mathbf{e}}{ }^{H} \mathbf{H}_{\mathbf{e}}$ and making all other matrices $\mathbf{A}_{i}$ to be (successively embedded) $i \times i$ principal submatrices of $\mathbf{H}_{\mathbf{e}}{ }^{H} \mathbf{H}_{\mathbf{e}}$, such that

3 Let $\mathcal{W}\left(n, \sum\right)$ denote a Wishart distribution with degree of freedom $n$ and covariance (also called scale) matrix $\sum$. The matrix $\mathbf{D}$ given by (12) follows a Wishart distribution since if $\mathbf{B}_{1} \in \mathcal{W}\left(n_{1}, \sum\right)$ and $\mathbf{B}_{2} \in \mathcal{W}\left(n_{2}, \sum\right)$ then $\mathbf{B}_{1}+\mathbf{B}_{2} \in \mathcal{W}\left(n_{1}+n_{2}, \sum\right)$. the smallest matrix is $\mathbf{A}_{M}=\mathbf{D}$. Then we repeatedly apply the first inequality in the Sturmian to get:

$$
\begin{gathered}
\lambda_{M L_{d}}\left(\mathbf{A}_{M L_{d}}\right) \leq \lambda_{M L_{d}-1}\left(\mathbf{A}_{M L_{d}-1}\right) \leq \cdots \leq \lambda_{M}\left(\mathbf{A}_{M}\right) \\
\lambda_{M L_{d}-1}\left(\mathbf{A}_{M L_{d}}\right) \leq \lambda_{M L_{d}-2}\left(\mathbf{A}_{M L_{d}-1}\right) \leq \cdots \leq \lambda_{M-1}\left(\mathbf{A}_{M}\right) \\
\vdots \\
\vdots \\
\lambda_{M L_{d}-M+1}\left(\mathbf{A}_{M L_{d}}\right) \leq \lambda_{M L_{d}-M}\left(\mathbf{A}_{M L_{d}-1}\right) \leq \cdots \leq \lambda_{1}\left(\mathbf{A}_{M}\right)
\end{gathered}
$$

This implies that the $M$ smallest eigenvalues of $\mathbf{H}_{\mathbf{e}}{ }^{H} \mathbf{H}_{\mathbf{e}}$ are bounded above, one by one, by the $M$ eigenvalues of $\mathbf{D}$. Hence:

$$
P_{\text {out }} \geqslant \mathbb{P}\left(\sum_{k=1}^{M} \frac{1}{\rho \lambda_{k}(\mathbf{D})}>M L_{d} 2^{-\frac{R}{M \alpha}}\right) .
$$

D is a sum of $(\nu+1)$ central Wishart matrices each with $N$ degrees of freedom and with identity covariance matrix, i.e. $\mathbf{D} \in \mathcal{W}((\nu+1) N, I)$.

$$
\begin{aligned}
& \mathbb{P}\left(\sum_{k=1}^{M} \frac{1}{\rho \lambda_{k}(\mathbf{D})}>M L_{d} 2^{-\frac{R}{M \alpha}}\right) \\
& =\mathbb{P}\left(\sum_{k=1}^{M} \rho^{-1} \lambda_{k}\left(\mathbf{D}^{-1}\right)>M L_{d} 2^{-\frac{R}{M \alpha}}\right) \\
& =\mathbb{P}\left(\sum_{k=1}^{M} \rho^{-1}\left[\mathbf{D}^{-1}\right]_{k k}>M L_{d} 2^{-\frac{R}{M \alpha}}\right) \\
& >\mathbb{P}\left(\rho^{-1}\left[\mathbf{D}^{-1}\right]_{k k}>M L_{d} 2^{-\frac{R}{M \alpha}}\right) \\
& \doteq \mathbb{P}\left(\frac{1}{\left[\mathbf{D}^{-1}\right]_{k k}}<\rho^{-1}\right)
\end{aligned}
$$

where (15) follows from the properties of eigenvalues, (16) follows because the trace is equal to the sum of eigenvalues, and (17) is true because $\sum_{k=1}^{M}\left[\mathbf{D}^{-1}\right]_{k k}>\left[\mathbf{D}^{-1}\right]_{k k}$. Here we use the fact that the Wishart matrix $\mathbf{D}$ and its inverse are positive definite, hence the diagonal elements are positive numbers [27].

Since $\mathbf{D} \in \mathcal{W}((\nu+1) N, I)$, each $\frac{1}{\left[\mathbf{D}^{-1}\right]_{k k}}$ obeys a chisquare distribution with $2(N(\nu+1)-M+1)$ degrees of freedom [28, Section III]. Hence the outage bound in (18) can be evaluated as follows

$$
\begin{aligned}
P_{\text {out }} & >\mathbb{P}\left(\frac{1}{\left[\mathbf{D}^{-1}\right]_{k k}}<\rho^{-1}\right) \\
& \doteq \rho^{-(N(\nu+1)-M+1)}
\end{aligned}
$$

where (19) follows as in [21], [23]. Thus the diversity is

$$
d_{\text {out }} \leq(N(\nu+1)-M+1)
$$

Remark 1: This upper bound shows that for a MIMO system with multiple antennas at both transmitter and receiver, the diversity of the ZF receiver under zero-padding is smaller than the $M N(\nu+1)$ diversity order achieved by the maximum likelihood receiver. This is in stark contrast with the SISO case, where it is known that the $\mathrm{ZF}$ receiver achieves the maximum spatial temporal diversity in the SISO channel $(M=N=1)[1]$. 
2) Diversity Lower Bound: A tight lower bound is obtained for the special case of $L_{d}=1$. In this case the channel matrix is given by

$$
\mathbf{H}_{\mathbf{e}}=\left[\begin{array}{c}
\mathbf{H}_{0} \\
\mathbf{H}_{1} \\
\vdots \\
\mathbf{H}_{\nu}
\end{array}\right] .
$$

Rewriting (9),

$$
\begin{aligned}
P_{\text {out }} & \doteq \mathbb{P}\left(\frac{1}{L} \sum_{k=1}^{M L_{d}} \log \frac{\rho}{\left[\left(\mathbf{H}_{\mathbf{e}}{ }^{H} \mathbf{H}_{\mathbf{e}}\right)^{-1}\right]_{k k}}<R\right) \\
& =\mathbb{P}\left(\frac{1}{L} \sum_{k=1}^{M L_{d}} \log \rho^{-1}\left[\left(\mathbf{H}_{\mathbf{e}}{ }^{H} \mathbf{H}_{\mathbf{e}}\right)^{-1}\right]_{k k}>-R\right) \\
& =\mathbb{P}\left(\prod_{k=1}^{M L_{d}} \rho^{-1}\left[\left(\mathbf{H}_{\mathbf{e}}{ }^{H} \mathbf{H}_{\mathbf{e}}\right)^{-1}\right]_{k k}>2^{-R L}\right) \\
& \leqslant \mathbb{P}\left(\frac{1}{M L_{d}} \sum_{k=1}^{M L_{d}} \rho^{-1}\left[\left(\mathbf{H}_{\mathbf{e}}{ }^{H} \mathbf{H}_{\mathbf{e}}\right)^{-1}\right]_{k k}>2^{-\frac{R L}{M L_{d}}}\right) \\
& =\mathbb{P}\left(\frac{1}{M L_{d}} \sum_{k=1}^{M L_{d}} \frac{1}{\rho \lambda_{k}}>2^{-\frac{R L}{M L_{d}}}\right) \\
& \leqslant \mathbb{P}\left(\frac{1}{\rho \lambda_{\min }}>2^{-\frac{R L}{M L_{d}}}\right) \\
& \doteq \mathbb{P}\left(\lambda_{\min }<\rho^{-1}\right)
\end{aligned}
$$

where (23) follows from Jensen's inequality. When $L_{d}=1$ the matrix $\mathbf{H}_{\mathbf{e}} \in \mathcal{W}((\nu+1) N, I)$. The minimum eigenvalue $\lambda_{\min }$ has distribution that is proportional to $\lambda^{N(\nu+1)-M}$ near zero [29], [30]. Thus the probability in (24) can be evaluated similarly to $[14$, Section III] to obtain

$$
\mathbb{P}\left(\lambda_{\min }<\rho^{-1}\right) \doteq \rho^{-(N(\nu+1)-M+1)}
$$

Therefore we have shown

$$
d_{\text {out }} \geq(N(\nu+1)-M+1)
$$

Comparing with the upper bound obtained earlier, we see that when $L_{d}=1$ the bounds are tight and we have $d_{\text {out }}=(N(\nu+$ 1) $-M+1)$.

Remark 2: The lower bound for $L_{d}>1$ remains an open problem.

\section{The Zero PAdDing MMSE Receiver}

For this system upper and lower bounds on diversity were obtained in [25] that were not tight against each other except for the case $L_{d}=1$. In this section we provide a new lower bound that is tight against the existing upper bound and thus completes the characterization of the diversity of zero-padding MMSE MIMO receiver. We start with a brief sketch of the proof of the diversity upper bound from [25], since partial intermediate results from it will be used for the subsequent analyses. The reader is invited to consult [25] for full details of this result.
3) Diversity Upper Bound: Using the MMSE SINR given in (5), the outage probability is given by

$$
\begin{aligned}
P_{\text {out }} & =\mathbb{P}\left(\frac{1}{L_{d}} \sum_{k=1}^{M L_{d}} \log \left(1+\gamma_{k}\right)<R\right) \\
& =\mathbb{P}\left(\frac{1}{L_{d}} \sum_{k=1}^{M L_{d}} \log \frac{1}{\left(\mathbf{I}+\rho \mathbf{H}_{\mathbf{e}}{ }^{H} \mathbf{H}_{\mathbf{e}}\right)_{k k}^{-1}}<R\right) \\
& \geqslant \mathbb{P}\left(M \log \frac{1}{M L_{d}} \sum_{k=1}^{M L_{d}} \frac{1}{\left(\mathbf{I}+\rho \mathbf{H}_{\mathbf{e}}{ }^{H} \mathbf{H}_{\mathbf{e}}\right)_{k k}^{-1}}<M L_{d} 2^{\frac{R}{M}}\right)
\end{aligned}
$$

where we have used Jensen's inequality to obtain (28). Define

$$
\mathbf{D}=\sum_{i=0}^{\nu} \mathbf{H}_{i}^{H} \mathbf{H}_{i}
$$

From [25, Section VI.B.1],

$$
\begin{aligned}
\mathbb{P}\left(\sum_{k=1}^{M L_{d}}\right. & \left.\frac{1}{\left(\mathbf{I}+\rho \mathbf{H}_{\mathbf{e}}{ }^{H} \mathbf{H}_{\mathbf{e}}\right)_{k k}^{-1}}<M L_{d} 2^{\frac{R}{M}}\right) \\
& \doteq \mathbb{P}\left(\sum_{k=1}^{M} \frac{1}{1+\rho \lambda_{k}(\mathbf{D})}>\left\lceil M 2^{-\frac{R}{M}}\right\rceil\right) \\
& \doteq \rho^{-d^{*}}
\end{aligned}
$$

where

$$
d^{*}=\left\lceil M 2^{-\frac{R}{M}}\right\rceil^{2}+|(\nu+1) N-M|\left\lceil M 2^{-\frac{R}{M}}\right\rceil
$$

and we have been able to take the last step in the same manner as the analogous result in the flat fading channel [25, Section II] because $\mathbf{D}$ is a sum of $(\nu+1)$ central Wishart matrices each with $N$ degrees of freedom and with identity covariance matrix, i.e. $\mathbf{D} \in \mathcal{W}((\nu+1) N, I)$.

4) Diversity Lower Bound: We now proceed to provide the new diversity lower bound. We use a bounding technique similar to [31]. We first provide the sketch of the proof and then proceed with the details. Define the event $\mathcal{J}$

$$
\mathcal{J} \triangleq\left\{\sum_{k=1}^{M} \frac{1}{1+\rho \lambda_{k}(\mathbf{D})}>\left\lceil M 2^{-\frac{R}{M}}\right\rceil\right\}
$$

Expressions (27) through (30) imply:

$$
P_{\text {out }} \geqslant P(\mathcal{J}) \text {. }
$$

The major steps in the proof involve showing that error probability $P(E)$ is bounded as follows

$$
P(E) \leqslant P(\mathcal{J}) .
$$

and also that using Fano inequality [21], we have

$$
P(E) \geqslant P_{\text {out }}
$$

Thus, using (33), (34) and (35),

$$
\mathbb{P}(\mathcal{J}) \dot{\leqslant} P_{\text {out }} \leqslant P(E) \leqslant \mathbb{P}(\mathcal{J})
$$

which establishes that

$$
\begin{aligned}
P_{\text {out }} & \doteq \mathbb{P}(\mathcal{J}) \\
P(E) & \doteq \mathbb{P}(\mathcal{J})
\end{aligned}
$$


We now proceed with the details. We want to show that $P_{\text {err }} \leqslant P(\mathcal{J})$. Using Bayes Theorem

$$
\begin{aligned}
P(E) & =\mathbb{P}(E \mid \mathcal{J}) \mathbb{P}(\mathcal{J})+\mathbb{P}(E, \overline{\mathcal{J}}) \\
& \leqslant \quad \mathbb{P}(\mathcal{J})+\mathbb{P}(E, \overline{\mathcal{J}}) \\
& \doteq \quad \rho^{-d^{*}}+\mathbb{P}(E, \overline{\mathcal{J}})
\end{aligned}
$$

where we have used (31) to obtain (39). We will now show

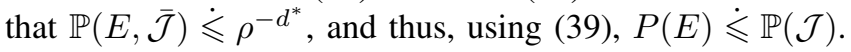

The error probability can be bounded via the pairwise error probability and the union bound. The pairwise error probability subject to a channel realization $\mathbf{H}_{\mathbf{e}}$ can be computed in a manner similar to [25, Section IV-A]

$$
\begin{aligned}
\mathbb{P}\left[s_{k} \rightarrow s_{j} \mid \mathbf{H}_{\mathbf{e}}=H\right] & \dot{\leqslant} 4 e^{-\frac{\rho}{\sigma_{\tilde{\mathbf{n}}}^{2}(k)}} \\
& \leqslant 4 e^{-\left(\sum_{k=1}^{L} \frac{\rho \lambda_{k}}{\left(1+\rho \lambda_{k}\right)^{2}}\right)^{-1}}
\end{aligned}
$$

where $\sigma_{\tilde{\mathbf{n}}}^{2}(k)$ is the variance of the interference plus noise. Using union bound for codewords of length $l$ we get

$$
\mathbb{P}\left(E \mid \mathbf{H}_{\mathbf{e}}=H\right) \dot{\leqslant} 2^{R l} e^{-\left(\sum_{k=1}^{L} \frac{\rho \lambda_{k}}{\left(1+\rho \lambda_{k}\right)^{2}}\right)^{-1}}
$$

Now define $\left\{\alpha_{k}\right\}$ as the exponential orders of the eigenvalues of $\mathbf{H}_{e}^{H} \mathbf{H}_{e}$, i.e., $\lambda_{k} \triangleq \rho^{-\alpha_{k}}$. This is a useful change of variable that is by now fairly common in diversity analysis, see e.g. [21]. Thus we have:

$$
\begin{aligned}
\sum_{k=1}^{M L_{d}} \frac{1}{1+\rho \lambda_{k}} & \doteq \sum_{\alpha_{k}>1} 1+\sum_{\alpha_{k}<1} \rho^{\alpha_{k}-1} \\
& \doteq M(\boldsymbol{\alpha})+\max _{\left\{\alpha_{k}: \alpha_{k}<1\right\}} \rho^{\alpha_{k}-1} \\
& \doteq M(\boldsymbol{\alpha})
\end{aligned}
$$

where $M(\boldsymbol{\alpha}) \triangleq \sum_{\alpha_{k}>1} 1$. Similarly, we can define $\lambda_{k}(\mathbf{D}) \triangleq$ $\rho^{-\tilde{\alpha}_{k}}$ we have

$$
\sum_{k=1}^{M} \frac{1}{1+\rho \lambda_{k}(\mathbf{D})} \doteq M(\tilde{\boldsymbol{\alpha}})
$$

where $M(\tilde{\boldsymbol{\alpha}}) \triangleq \sum_{\tilde{\alpha}_{k}>1} 1$.

Now consider two sets: $\{\boldsymbol{\alpha}: M(\boldsymbol{\alpha})=0\}$ and its complement. We can evaluate $\mathbb{P}(E, \overline{\mathcal{J}})$ by computing

$$
\begin{aligned}
\mathbb{P}(E, \overline{\mathcal{J}}) & =\int_{M(\boldsymbol{\alpha})=0} \mathbb{P}(E, \overline{\mathcal{J}} \mid M(\boldsymbol{\alpha})=0) \mathbb{P}(\boldsymbol{\alpha}) \mathrm{d} \boldsymbol{\alpha} \\
& +\int_{M(\boldsymbol{\alpha}) \geqslant 1} \mathbb{P}(E, \overline{\mathcal{J}} \mid M(\boldsymbol{\alpha}) \geqslant 1) \mathbb{P}(\boldsymbol{\alpha}) \mathrm{d} \boldsymbol{\alpha}
\end{aligned}
$$

We have:

$$
\begin{aligned}
\mathbb{P}(E, \overline{\mathcal{J}} \mid M(\boldsymbol{\alpha})=0) & =\mathbb{P}(E \mid \overline{\mathcal{J}}, M(\boldsymbol{\alpha})=0) \mathbb{P}(\overline{\mathcal{J}}) \\
& \leqslant \mathbb{P}(E \mid \overline{\mathcal{J}}, M(\boldsymbol{\alpha})=0)
\end{aligned}
$$

where (46) follows since we have $\mathbb{P}(\overline{\mathcal{J}})=1-\mathbb{P}(\mathcal{J}) \doteq 1-$ $\rho^{-d^{*}} \doteq 1$ (cf. Eq. (31)).

We now evaluate $\mathbb{P}(E \mid \overline{\mathcal{J}}, M(\boldsymbol{\alpha})=0)$. Define $M_{S}$ to be the set of the $M$ smallest eigenvalues of $\mathbf{H}_{\mathbf{e}}{ }^{H} \mathbf{H}_{\mathbf{e}}$. Then we have

$$
\sum_{k=1}^{M L_{d}} \frac{1}{1+\rho \lambda_{k}} \geqslant \sum_{\lambda_{k} \in M_{S}} \frac{1}{1+\rho \lambda_{k}}
$$

$$
\geqslant \sum_{k=1}^{M} \frac{1}{1+\rho \lambda_{k}(\mathbf{D})}
$$

where (47) follows using the Sturmian separation theorem as in Section IV-3. Similarly to [25, Eq.(56)], it can be shown that

$$
\mathbb{P}(E \mid \overline{\mathcal{J}}, M(\boldsymbol{\alpha})=0) \dot{\leqslant} \rho^{-M N(\nu+1)}
$$

In the same manner, in the complementary region $\{\boldsymbol{\alpha}$ : $M(\boldsymbol{\alpha}) \geqslant 1\}$ we have

$$
\begin{aligned}
\mathbb{P}(E, \overline{\mathcal{J}} \mid M(\boldsymbol{\alpha}) \geqslant 1) & =\mathbb{P}(E \mid \overline{\mathcal{J}}, M(\boldsymbol{\alpha}) \geqslant 1) \mathbb{P}(\overline{\mathcal{J}}) \\
& \leqslant \mathbb{P}(E \mid \overline{\mathcal{J}}, M(\boldsymbol{\alpha}) \geqslant 1)
\end{aligned}
$$

Recall that

$$
\begin{aligned}
& \mathbb{P}\left(E \mid \mathbf{H}_{\mathbf{e}}=H\right) \\
& \dot{\leqslant} 2^{R l} e^{-\left(\sum_{k=1}^{L} \frac{\rho \lambda_{k}}{\left(1+\rho \lambda_{k}\right)^{2}}\right)^{-1}} \\
& <e^{2^{R l}} e^{-\left(\sum_{k} \frac{1}{1+\rho \lambda}-\sum_{k} \frac{1}{\left(1+\rho \lambda_{k}\right)^{2}}\right)^{-1}} \\
& =e^{2^{R l}} e^{-\left(\sum_{k} \frac{1}{1+\rho \lambda_{k}}\right)^{-1}} \\
& \times \exp \left[-\frac{\sum_{k} \frac{1}{\left(\rho \lambda_{k}+1\right)^{2}}}{\left(\sum_{k} \frac{1}{1+\rho \lambda_{k}}\right)\left(\sum_{k} \frac{\rho \lambda_{k}}{\left(1+\rho \lambda_{k}\right)^{2}}\right)}\right] \\
& \leqslant e^{2^{R l}} e^{-\left(\frac{M L_{d}-M}{M L_{d}}+\frac{1}{M L_{d}} \sum_{k=1}^{M} \frac{1}{1+\rho \lambda_{k}}\right)^{-1}} \\
& \times \exp \left[-\frac{\frac{1}{M L_{d}} \sum_{k} \frac{1}{\left(\rho \lambda_{k}+1\right)^{2}}}{\left(\frac{1}{M L_{d}} \sum_{k} \frac{1}{1+\rho \lambda_{k}}\right)\left(\sum_{k} \frac{\rho \lambda_{k}}{\left(1+\rho \lambda_{k}\right)^{2}}\right)}\right] \\
& \leqslant e^{2^{R l}} e^{-\left(\frac{M L_{d}-M}{M L_{d}}+\frac{1}{M L_{d}} \sum_{k=1}^{M} \frac{1}{1+\rho \lambda_{k}}\right)^{-1}} \\
& \times \exp \left[-\frac{\frac{1}{M L_{d}} \sum_{k} \frac{1}{\left(\rho \lambda_{k}+1\right)^{2}}}{\left(\frac{1}{M L_{d}} \sum_{k} \frac{1}{1+\rho \lambda_{k}}\right)\left(\sum_{k} \frac{\rho \lambda_{k}}{\left(1+\rho \lambda_{k}\right)^{2}}\right)}\right]
\end{aligned}
$$

where (51) follows by setting the $M L_{d}-M$ smallest eigenvalues to zero, (52) follows because the $M$ largest eigenvalues $\left\{\lambda_{K}\right\}$ are bounded below by the $M$ eigenvalues $\tilde{\lambda}_{k}$. By conditioning on $\overline{\mathcal{J}}$,

$$
\begin{aligned}
\mathbb{P}(E \mid \overline{\mathcal{J}}) \leqslant & e^{2^{R l}} e^{-\left(\frac{M L_{d}-M}{M L_{d}}+\frac{1}{M L_{d}} 2^{-\frac{R}{M}}\right)^{-1}} \\
& \times \exp \left[-\frac{\frac{1}{M L_{d}} \sum_{k} \frac{1}{\left(\rho \lambda_{k}+1\right)^{2}}}{\left(\frac{1}{M L_{d}} \sum_{k} \frac{1}{1+\rho \lambda_{k}}\right)\left(\sum_{k} \frac{\rho \lambda_{k}}{\left(1+\rho \lambda_{k}\right)^{2}}\right)}\right] \\
\doteq & \exp \left[-\frac{\frac{1}{M L_{d}} \sum_{k} \frac{1}{\left(\rho \lambda_{k}+1\right)^{2}}}{\left(\frac{1}{M L_{d}} \sum_{k} \frac{1}{1+\rho \lambda_{k}}\right)\left(\sum_{k} \frac{\rho \lambda_{k}}{\left(1+\rho \lambda_{k}\right)^{2}}\right)}\right]
\end{aligned}
$$

where the last step follows because the first and second exponentials in (53) are independent of $\rho$. We can now further condition on $M(\boldsymbol{\alpha}) \geqslant 1$ to get, via a technique similar to [25, Eq.(58)]:

$$
\begin{aligned}
\mathbb{P}(E \mid \overline{\mathcal{J}}, M(\boldsymbol{\alpha}) \geqslant 1) & \dot{\leqslant} \exp \left(\rho^{1-\max _{k} \alpha_{k}}\right) \\
& \dot{\leqslant} \rho^{-M N(\nu+1)} .
\end{aligned}
$$

We now use (48) and (56) to bound (45)

$\mathbb{P}(E, \overline{\mathcal{J}})$ 


$$
\begin{aligned}
= & \int_{M(\boldsymbol{\alpha})=0} \mathbb{P}(E, \overline{\mathcal{J}} \mid M(\boldsymbol{\alpha})=0) \mathbb{P}(\boldsymbol{\alpha}) \mathrm{d} \boldsymbol{\alpha} \\
& +\int_{M(\boldsymbol{\alpha}) \geqslant 1} \mathbb{P}(E, \overline{\mathcal{J}} \mid M(\boldsymbol{\alpha}) \geqslant 1) \mathbb{P}(\boldsymbol{\alpha}) \mathrm{d} \boldsymbol{\alpha} \\
\dot{\leqslant} & \int_{M(\boldsymbol{\alpha})=0} \rho^{-M N(\nu+1)} \mathbb{P}(\boldsymbol{\alpha}) \mathrm{d} \boldsymbol{\alpha} \\
& +\int_{M(\boldsymbol{\alpha}) \geqslant 1} \rho^{-M N(\nu+1)} \mathbb{P}(\boldsymbol{\alpha}) \mathrm{d} \boldsymbol{\alpha} \\
= & \rho^{-M N(\nu+1)}\left[\int_{M(\boldsymbol{\alpha})=0} \mathbb{P}(\boldsymbol{\alpha}) \mathrm{d} \boldsymbol{\alpha}+\int_{M(\boldsymbol{\alpha}) \geqslant 1} \mathbb{P}(\boldsymbol{\alpha}) \mathrm{d} \boldsymbol{\alpha}\right] \\
= & \rho^{-M N(\nu+1)} .
\end{aligned}
$$

Using (57), we can now bound $P(E)$ in (39)

$$
\begin{aligned}
P(E) & \leqslant \rho^{-d^{*}}+\mathbb{P}(E, \overline{\mathcal{J}}) \\
& \leqslant \rho^{-d^{*}}+\rho^{-M N(\nu+1)} \\
& \doteq \rho^{-d^{*}}
\end{aligned}
$$

which establishes (34) and hence (36) and (37).

\section{LAtTice Reduction Aided Equalization}

The main problem with zero-forcing equalization, which also manifests itself in the system diversity, is noise enhancement. When $\mathbf{H}_{\mathbf{e}}$ is near-singular, zero-forcing amplifies the noise strongly along the smaller eigenvectors of the channel, causing difficulties for the detector. One way to address this problem is Lattice Reduction (LR) [3], [4]. In this section we show that lattice reduction aided equalization achieves full diversity. We begin with a short review of LR-aided detection.

The orthogonality of a matrix $\mathbf{H}$ can be quantified using the notion of orthogonality defect defined as [32]

$$
\delta=\frac{\left(\left\|\mathbf{b}_{1}\right\|^{2}\left\|\mathbf{b}_{2}\right\|^{2} \ldots\left\|\mathbf{b}_{M L_{d}}\right\|^{2}\right)}{\operatorname{det} \mathbf{H}^{H} \mathbf{H}}
$$

where $\mathbf{H}=\left[\mathbf{b}_{1} \ldots \mathbf{b}_{M L_{d}}\right]$. Moreover, the orthogonality defect can be bounded as follows [32]

$$
1 \leqslant \delta \leqslant c=2^{2 M L_{d}\left(M L_{d}-1\right)} .
$$

If the matrix $\mathbf{H}$ has orthogonal columns then $\mathbf{H}^{H} \mathbf{H}$ is diagonal and thus using ZF equalizer yields same performance as the ML detector, since the decision regions in both cases are the same. However, since $\mathbf{H}$ is in general not orthogonal, linear equalization does not perform as well as ML.

Lattice reduction finds a mapping of the lattice onto itself so that from the viewpoint of the detector, the columns of the transformed equivalent channel are near-orthonormal. More specifically, lattice reduction finds a unimodular ${ }^{4}$ matrix $\mathbf{T}$ such that $\mathbf{H}=\mathbf{H}_{\mathrm{e}} \mathbf{T}$ is approximately orthogonal. ${ }^{5}$ The

\footnotetext{
${ }^{4} \mathrm{~A}$ unimodular matrix is a matrix that has the following property: the entries of both the matrix and its inverse are complex integers and $\operatorname{det}(\mathbf{T})= \pm 1$ or $\pm j$.

${ }^{5}$ Various algorithms exist for efficiently finding this transformation, among them the LLL algorithm [33].
}

process is as follows:

$$
\mathbf{y}=\mathbf{H}_{\mathrm{e}} \mathbf{x}+\mathbf{n}=\left(\mathbf{H}_{\mathbf{e}} \mathbf{T}\right) \underbrace{\mathbf{T}^{-1} \mathbf{x}}_{\hat{\mathbf{x}}}+\mathbf{n}
$$

Denoting with $\mathbf{W}_{Z F}$ the $\mathrm{ZF}$ equalizer for the reduced channel $\mathbf{H}=\mathbf{H}_{\mathbf{e}} \mathbf{T}$, we have

$$
\mathbf{z}=\mathbf{W}_{Z F} \mathbf{y}=\hat{\mathbf{x}}+\mathbf{W}_{Z F} \mathbf{n}
$$

Now $\hat{\mathbf{x}}$ is decoded and $\mathbf{x}$ is recovered by multiplying with $\mathbf{T}$.

Lattice reduction aided detection has been proposed and analyzed in several scenarios. A quantitative analysis was presented in [3] where it is shown that the error rate performance of LR is within $3 \mathrm{~dB}$ of ML for a $2 \times 2$ Gaussian MIMO channel model. The LR-aided detection (LRAD) is known to achieve full receive diversity in uncoded flat MIMO broadcast channel [6]. It is also shown [7] that LRAD ( with its modified version complex LLL [34]) achieves full receive diversity in MIMO flat V-BLAST channel. LRAD was also studied for OFDM SISO channel [35]. The diversity of lattice-reductionaided receivers in the frequency-selective MIMO channel has been an open problem, which is addressed in this section.

\section{A. Diversity Analysis}

An $n$-dimensional lattice $\mathcal{L}$ in the $m$-dimensional space is generated by linear combination of $n$ linearly independent vectors $\left\{\mathbf{b}_{i}\right\}$

$$
\mathcal{L}=\left\{\sum_{i=1}^{n} x_{i} \mathbf{b}_{i} \mid x_{i} \in \mathbb{Z}, \mathbf{b}_{i} \in \mathbb{C}^{m}\right\}
$$

Each lattice can be represented by (infinitely) many different bases. Lenstra et al. [33] proposed the first polynomial time algorithm (LLL) that finds a near-orthogonal basis whose vectors are all roughly the same size, specifically, the ratio of the second norm of any two vectors of the basis is no bigger than $2^{(n-1) / 2}$. The LLL algorithm was originally introduced for real lattice bases and was shown to require $O\left(n^{4}\right)$ arithmetic operations. Complex LLL (CLLL) algorithms have been proposed in [7], [34] that yield similar performance as LLL when applied to complex channel but with reduced complexity.

We start with outlining a recent result from [36] which characterizes the performance of lattice-reduction-aided detection in infinite lattice decoding (ILD). Let $d_{I L D}$ denote the Euclidean distance between a lattice point to the closest boundary of the corresponding Voronoi cell, and $d_{Z F}$ denote the distance between the same lattice point and the closest boundary of the decision region of the ZF detector (cf. Figure 2). The term "proximity factor" is defined in [36] as follows

$$
\kappa_{z f} \triangleq \sup _{\mathbf{H} \in \mathcal{H}_{\text {reduced }}} \frac{d_{I L D}^{2}}{d_{Z F}^{2}}
$$

where the supremum is taken over the set $\mathcal{H}_{\text {reduced }}$ of basis matrices satisfying a certain reduction criterion.

The factor $\kappa_{z f}$ is a function of not only the lattice, but also the basis that is used to represent it, and without lattice reduction $\kappa_{z f}$ is unbounded. With reduction, this factor is upper bounded by a constant that is a function of the cardinality of 


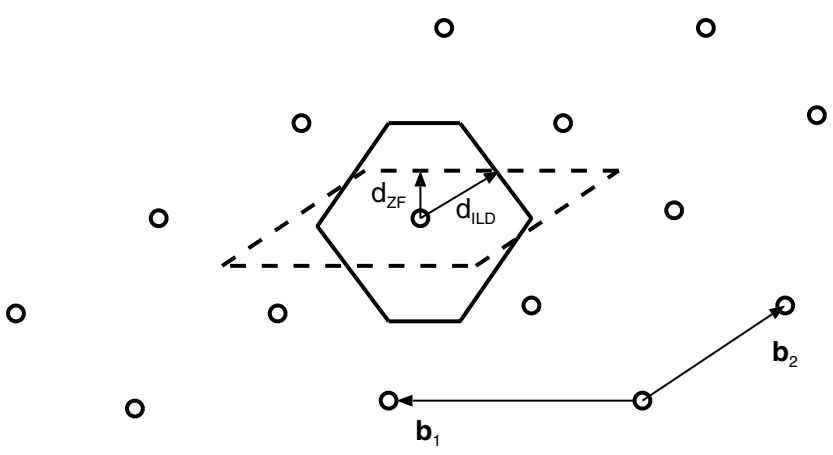

Fig. 2. Decision regions of ZF (dashed) and infinite lattice decoding (solid) with the corresponding minimum distances in a 2-dimensional lattice.

the basis of the lattice [36]. Although [36] is based on a real channel model, the analysis is easily extended to the complex case by rewriting the system model in (61) as

$$
\left[\begin{array}{l}
\operatorname{Re}\{\mathbf{y}\} \\
\operatorname{Im}\{\mathbf{y}\}
\end{array}\right]=\left[\begin{array}{rr}
\operatorname{Re}\{\mathbf{H}\} & -\operatorname{Im}\{\mathbf{H}\} \\
\operatorname{Im}\{\mathbf{H}\} & \operatorname{Re}\{\mathbf{H}\}
\end{array}\right]\left[\begin{array}{l}
\operatorname{Re}\{\mathbf{x}\} \\
\operatorname{Im}\{\mathbf{x}\}
\end{array}\right]+\left[\begin{array}{l}
\operatorname{Re}\{\mathbf{n}\} \\
\operatorname{Im}\{\mathbf{n}\}
\end{array}\right]
$$

Similar to the analysis of [36], by using (63) for a complex lattice it can be shown that the error probability of the LRaided $\mathrm{ZF}$ detector is upper bounded as

$$
P_{e, \mathrm{ZF}} \leqslant 2 M L_{d} P_{e}
$$

where $P_{e}$ is the error probability of the infinite lattice decoding. It is known that for sufficiently large block lengths the outage and error probabilities decay at the same rate with increasing SNR and we thus have [21], [37]

$$
P_{e} \doteq P_{\text {out }} \text {. }
$$

Therefore we concentrate on outage calculations. The outage probability $P_{\text {out }}$ is given by

$$
P_{\text {out }} \triangleq \mathbb{P}\left(\frac{1}{M L_{d}} I(\mathbf{x} ; \mathbf{y})<R\right)
$$

where $I(\mathbf{x} ; \mathbf{y})=\log \operatorname{det}\left(\mathbf{I}+\rho^{-1} \mathbf{H}_{e}^{H} \mathbf{H}_{e}\right)$ [38]. We now bound $\operatorname{det}\left(\mathbf{I}+\rho^{-1} \mathbf{H}_{e}^{H} \mathbf{H}_{e}\right)$

$$
\begin{aligned}
\operatorname{det}\left(\mathbf{I}+\rho^{-1} \mathbf{H}_{e}^{H} \mathbf{H}_{e}\right) & =\prod_{k=1}^{M L_{d}}\left(1+\rho \lambda_{k}\right) \\
& \geqslant 1+\rho \lambda_{\max } \\
& \geqslant 1+\rho \frac{1}{M L_{d}} \sum_{k=1}^{M L_{d}} \lambda_{k}
\end{aligned}
$$

where $\left\{\lambda_{k}\right\}$ are the eigenvalues of $\mathbf{H}_{e}^{H} \mathbf{H}_{e}$. We can thus bound the outage probability in (66)

$$
\begin{aligned}
P_{\text {out }} & \leqslant \mathbb{P}\left(\frac{1}{M L_{d}} \log \left(1+\rho \frac{1}{M L_{d}} \sum_{k=1}^{M L_{d}} \lambda_{k}\right)<R\right) \\
& =\mathbb{P}\left(\rho \frac{1}{M L_{d}} \sum_{k=1}^{M L_{d}} \lambda_{k}<2^{R M L_{d}}-1\right) \\
& =\mathbb{P}\left(\rho \sum_{k=1}^{M L_{d}} \lambda_{k}<M L_{d}\left(2^{R M L_{d}}-1\right)\right)
\end{aligned}
$$

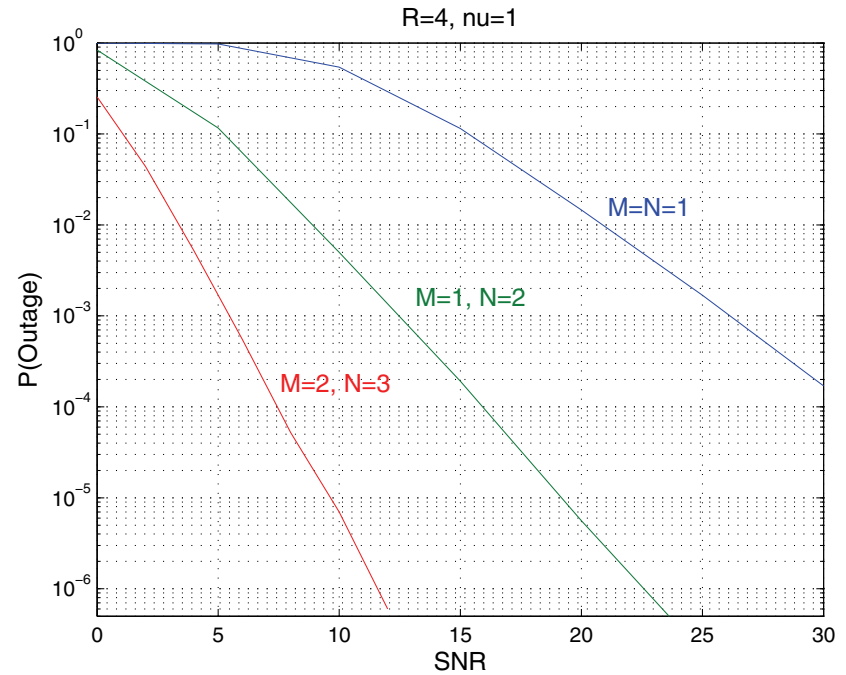

Fig. 3. Outage probability of ZF receiver, 2-tap channel, $L_{d}=3$ and $R=4$ $\mathrm{bps} / \mathrm{Hz}$.

$$
\doteq \mathbb{P}\left(\sum_{k=1}^{M L_{d}} \lambda_{k}<\rho^{-1}\right)
$$

Recall from the definition of the Frobenius norm

$$
\left\|\mathbf{H}_{\mathbf{e}}\right\|_{F}^{2}=\sqrt{\sum_{i, j}\left|h_{i j}\right|^{2}}=\sqrt{\operatorname{tr}\left(\mathbf{H}_{\mathbf{e}}{ }^{H} \mathbf{H}_{\mathbf{e}}\right)}=\sqrt{\sum_{k=1}^{M L_{d}} \lambda_{k}}
$$

where $h_{i, j}$ is the entry $(i, j)$ of the matrix $\mathbf{H}_{\mathbf{e}}$. Since $\mathbf{H}_{\mathbf{e}}$ is block Toeplitz it can be easily verified that

$$
\sum_{i, j}\left|h_{i j}\right|^{2}=L_{d} \sum_{m=1}^{M} \sum_{n=1}^{N} \sum_{k=0}^{\nu}\left|h_{m n, k}\right|^{2}
$$

Using (68) and (69) we have

$$
P_{\text {out }} \leqslant \mathbb{P}\left(L_{d} \sum_{m, n, k}\left|h_{m n, k}\right|^{2}<\rho^{-1}\right)
$$

Since $\sum_{m, n, k}\left|h_{m n, k}\right|^{2}$ is a chi-square random variable with $2 M N(\nu+1)$ degree of freedom, evaluating (70) yields [21], [39]

$$
P(\mathcal{O}) \leqslant \rho^{-M N(\nu+1)} .
$$

Thus the outage diversity of the LR-aided ZF is lower bounded $d_{\text {out }} \geq M N(\nu+1)$. We also know $d_{\text {out }} \leq$ $M N(\nu+1)$ via the diversity of the optimum ML receiver. Therefore we conclude that the LR-aided ZF outage diversity is $d_{\text {out }}^{L R}=M N(\nu+1)$.

Remark 3: We note that the identical proof easily follows for the MMSE equalizer, therefore it is established that the lattice-reduction MMSE equalizer also enjoys full diversity.

\section{Simulation}

Monte Carlo simulations are provided for several MIMO configurations. The simulations offered in this section are carefully chosen because high diversity orders can appear 


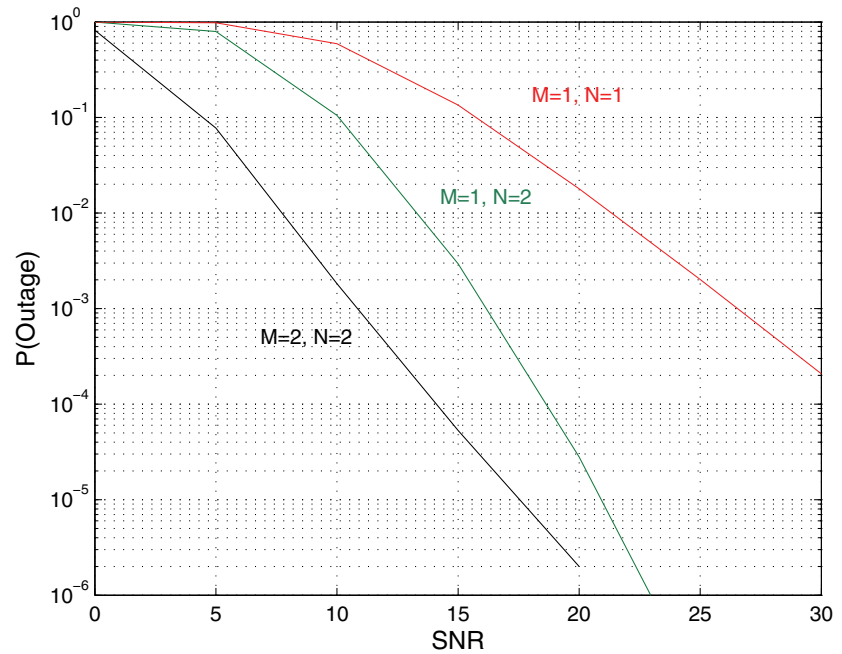

Fig. 4. Outage probability of MMSE receiver, 2-tap channel and $R=4$ bps/Hz.

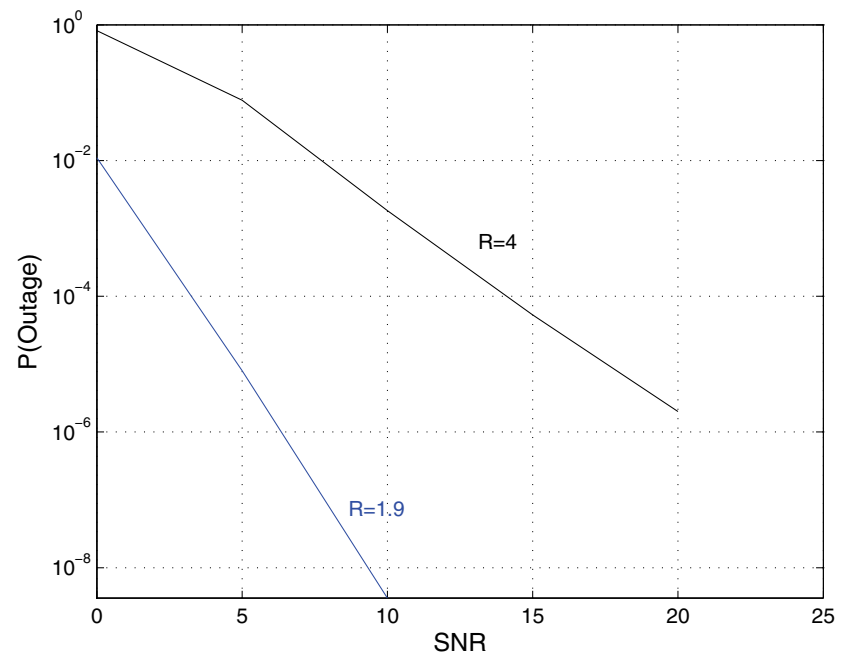

Fig. 5. Outage probability of MMSE receiver, 2-tap channel and $M=N=$

in frequency selective channels that are difficult to demonstrate via simulations. Figure 3 shows the outage probabilities of ZF MIMO receiver under zero-padding transmission for $M=N=1, M=1, N=2$ and $M=2, N=3$ with 2 channel taps and $R=4 \mathrm{~b} / \mathrm{s} / \mathrm{Hz}$. The diversities are respectively given by 2,4 and 5 . Figure 4 shows the outage probabilities of MMSE MIMO receiver under zero-padding transmission for $R=4 \mathrm{~b} / \mathrm{s} / \mathrm{Hz}$ and different antenna configurations. When $M=N=2$ the MMSE receiver diversity is 3 whereas when $M=1$ and $N=2$ the MMSE receiver diversity is 4. Figure 5 shows the outage probabilities of MMSE MIMO receiver for different values of $R$. From (32) the diversity at $R=1.9$ and $4 \mathrm{~b} / \mathrm{s} / \mathrm{Hz}$ is 8 and 3 respectively. Large diversities are not easily verified in simulations, but it is shown in Figure 5 that at $R=1.9 \mathrm{~b} / \mathrm{s} / \mathrm{Hz}$ the diversity is much larger than the diversity at $R=4 \mathrm{~b} / \mathrm{s} / \mathrm{Hz}$. Figure 6 shows the outage probabilities of lattice-reduction aided ZF MIMO

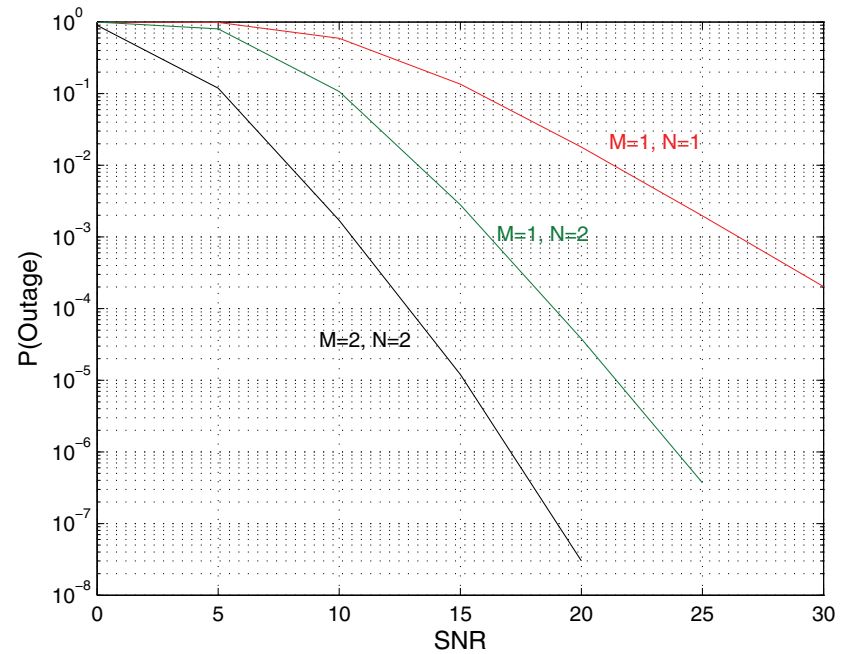

Fig. 6. Outage probability of LR-aided ZF receiver, 2-tap channel and $R=4$ bps/Hz.

receiver under zero-padding transmission for $M=N=1$, $M=1, N=2$ and $M=N=2$ with 2 channel taps and $R=4 \mathrm{~b} / \mathrm{s} / \mathrm{Hz}$.

\section{CONCLUSION}

This paper provides performance analysis of zero-forcing (ZF) and MMSE MIMO receivers in frequency selective channels under zero-padding transmission. It is shown that the performance of the MIMO ZF receiver under these conditions is generally highly suboptimal, even in the presence of outer coding across the data streams. However, in the special cases of the SISO channel, full diversity is achieved by the ZF receiver. The MMSE receiver provides better performance in a manner depending not only on antenna and channel configuration, but also on transmission rate. It is shown that the MMSE receiver attains optimal diversity at low rates. For improved performance, we investigate lattice-reduction aided equalization. It is shown that lattice-reduction-aided linear equalizers achieve the maximum spatial and temporal diversity at all spectral efficiencies.

\section{REFERENCES}

[1] C. Tepedelenlioglu, "Low complexity linear equalizers with maximum multipath diversity for zero-padded transmissions," in Proc. 2003 IEEE ICASSP, vol. 4, pp. 636-639.

[2] — , "Maximum multipath diversity with linear equalization in precoded OFDM systems," IEEE Trans. Inf. Theory, vol. 50, pp. 232-235, Jan. 2004.

[3] H. Yao and G. Wornell, "Lattice-reduction-aided detectors for MIMO communication systems," in Proc. 2002 IEEE GLOBECOM, vol. 1, pp. $424-428$.

[4] C. Windpassinger and R. Fischer, "Low-complexity near-maximumlikelihood detection and precoding for MIMO systems using lattice reduction," in Proc. 2003 IEEE ITA, pp. 345-348.

[5] C. Windpassinger, R. Fischer, and J. Huber, "Lattice-reduction-aided broadcast precoding," IEEE Trans. Commun., vol. 52, no. 12, pp. 20572060, Dec. 2004.

[6] M. Taherzadeh, A. Mobasher, and A. Khandani, "Communication over MIMO broadcast channels using lattice-basis reduction," IEEE Trans. Inf. Theory, vol. 53, no. 12, pp. 4567-4582, Dec. 2007. 
[7] X. Ma and W. Zhang, "Performance analysis for MIMO systems with lattice-reduction aided linear equalization," IEEE Trans. Commun., vol. 56, no. 2, pp. 309-318, Feb. 2008.

[8] D. Wübben, D. Seethaler, J. Jaldén, and G. Matz, "Lattice reduction," IEEE Signal Process. Mag., vol. 28, no. 3, pp. 70-91, May 2011.

[9] E. N. Onggosanusi, A. G. Dabak, T. Schmidl, and T. Muharemovic, "Capacity analysis of frequency-selective MIMO channels with suboptimal detectors," in Proc. 2002 IEEE ICASSP, vol. 3, pp. 2369-2372.

[10] A. Hedayat, A. Nosratinia, and N. Al-Dhahir, "Outage probability and diversity order of linear equalizers in frequency-selective fading channels," in Proc. 2004 Asilomar Conf. Signals, Syst. Comput., vol. 2, pp. 2032-2036.

[11] A. Tajer and A. Nosratinia, "Diversity order in ISI channels with singlecarrier frequency-domain equalizer," IEEE Trans. Wireless Commun., vol. 9, no. 3, pp. 1022-1032, Mar. 2010

[12] A. Tajer, A. Nosratinia, and N. Al-Dhahir, "Diversity order of infinitelength symbol-by-symbol linear equalization," EURASIP Special Issue Interference Management Wireless Commun. Syst., June 2010.

[13] D. Slock, "Diversity and coding gain of linear and decision-feedback equalizers for frequency-selective SIMO channels," in Proc. 2006 IEEE ISIT, pp. 605-609.

[14] K. R. Kumar, G. Caire, and A. L. Moustakas, "Asymptotic performance of linear receivers in MIMO fading channels," IEEE Trans. Inf. Theory, vol. 55 , no. 10 , pp. 4398-4418, Oct. 2009.

[15] A. Hesham Mehana and A. Nosratinia, "Diversity of MMSE MIMO receivers," in Proc. 2010 IEEE ISIT.

[16] A. Medles and D. Slock, "Optimal diversity vs multiplexing tradeoff for frequency selective MIMO channels," in Proc. 2005 IEEE ISIT, pp. 1813-1817.

[17] D. Slock, "Diversity-multiplexing tradeoff of simplified receivers for frequency-selective MIMO channels," in Proc. 2008 EURASIP European Sig. Proc. Conf.

[18] D. Wübben, R. Böhnke, V. Kühn, and K.-D. Kammeyer, "Nearmaximum-likelihood detection of MIMO systems using MMSE-based lattice reduction," in Proc. 2004 IEEE ICC, vol. 2, pp. 798-802.

[19] A. Scaglione, P. Stoica, S. Barbarossa, G. Giannakis, and H. Sampath, "Optimal designs for space-time linear precoders and decoders," IEEE Trans. Signal Process., vol. 50, no. 5, pp. 1051-1064, May 2002.

[20] H. Gao, P. J. Smith, and M. V. Clark, "Theoretical reliability of MMSE linear diversity combining in Rayleigh-fading additive interference channels," IEEE Trans. Commun., vol. 46, no. 5, pp. 666-672, May 1998.

[21] L. Zheng and D. N. C. Tse, "Diversity and multiplexing: a fundamental tradeoff in multiple-antenna channels," IEEE Trans. Inf. Theory, vol. 49, no. 5, pp. 1073-1096, May 2003.

[22] A. Hesham Mehana and A. Nosratinia, "The diversity of MMSE receiver over frequency-selective MIMO channel," in Proc. 2011 IEEE ISIT.

[23] L. Grokop and D. Tse, "Diversity-multiplexing tradeoff in ISI channels," IEEE Trans. Inf. Theory, vol. 55, no. 1, pp. 109-135, Jan. 2009.

[24] Z. Wang, X. Ma, and G. Giannakis, "Optimality of single-carrier zeropadded block transmissions," in Proc. 2002 IEEE WCNC, vol. 2, pp. 660-664.

[25] A. H. Mehana and A. Nosratinia, "Diversity of MMSE MIMO receivers," IEEE Trans. Inf. Theory, no. 10, pp. 6788-6805, Oct. 2012. Available: http://arxiv.org/abs/1102.1462

[26] I. Gradshteyn and I. Ryzbik, Tables of Integrals, Series, and Products, 6th ed. Academic Press, 2000.

[27] I. E. Telatar, "Capacity of multi-antenna Gaussian channels," European Trans. Telecommun., vol. 10, pp. 585-595, Nov./Dec. 1999.

[28] D. Gore, R. W. Heath, Jr., and A. Paulraj, "On performance of the zero forcing receiver in presence of transmit correlation," in Proc. 2002 IEEE ISIT, p. 159.

[29] E. Sengul, E. Akay, and E. Ayanoglu, "Diversity analysis of single and multiple beamforming," IEEE Trans. Commun., vol. 54, no. 6, pp. 990 993, June 2006
[30] Y. Jiang, M. K. Varanasi, and J. Li, "Performance analysis of ZF and MMSE equalizers for MIMO systems: an in-depth study of the high SNR regime," IEEE Trans. Inf. Theory, vol. 57, no. 4, pp. 2008-2026, Apr. 2011

[31] P. Coronel and H. Bölcskei, "Diversity-multiplexing tradeoff in selective-fading MIMO channels," in Proc. 2007 IEEE ISIT, pp. 28412845.

[32] M. Taherzadeh, A. Mobasher, and A. Khandani, "LLL reduction achieves the receive diversity in MIMO decoding," IEEE Trans. Inf. Theory, vol. 53, no. 12, pp. 4801-4805, Dec. 2007.

[33] A. K. Lenstra, H. W. Lenstra, and L. Lovász, "Factoring polynomials with rational coefficients," Mathematische Annalen, vol. 261, pp. 515534, 1982

[34] Y. H. Gan, C. Ling, and W. H. Mow, "Complex lattice reduction algorithm for low-complexity full-diversity MIMO detection," IEEE Trans. Signal Process., vol. 57, no. 7, pp. 2701-2710, July 2009.

[35] X. Ma, W. Zhang, and A. Swami, "Lattice-reduction aided equalization for OFDM systems," IEEE Trans. Wireless Commun., vol. 8, no. 4, pp. 1608-1613, Apr. 2009.

[36] C. Ling, "On the proximity factors of lattice reduction-aided decoding," IEEE Trans. Signal Process., vol. 59, no. 6, pp. 2795-2808, June 2011.

[37] Z. Wang and G. Giannakis, "A simple and general parameterization quantifying performance in fading channels," IEEE Trans. Commun., vol. 51, no. 8, pp. 1389-1398, Aug. 2003.

[38] N. Al-Dhahir and J. Cioffi, "Block transmission over dispersive channels: transmit filter optimization and realization, and MMSE-DFE receiver performance," IEEE Trans. Inf. Theory, vol. 42, no. 1, pp. 137160, Jan. 1996.

[39] A. Hedayat and A. Nosratinia, "Outage and diversity of linear receivers in flat-fading MIMO channels," IEEE Trans. Signal Process., vol. 55, no. 12 , pp. 5868-5873, Dec. 2007.

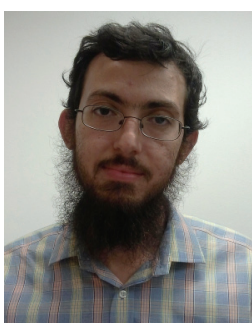

Ahmed Hesham Mehana (S'10) received his B.Sc. and M.Sc. degrees from Cairo University in 2004 and 2007, respectively, both in Electrical Engineering. He received the $\mathrm{Ph}$.D. degree in electrical engineering at the University of Texas at Dallas in 2012. $\mathrm{He}$ is currently with Samsung Telecommunication America. He was an intern at Research in Motion Co. Ltd during 2010 and research assistant in Texas A\&M at Qatar in 2008. His current interests include MIMO precoding, linear receivers, and interference management.

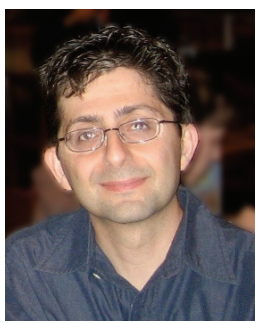

Aria Nosratinia (S'87-M'97-SM'04-F'10) is Erik Jonsson Distinguished Professor and associate head of the Electrical Engineering Department at the University of Texas at Dallas. He received his Ph.D. in Electrical and Computer Engineering from the University of Illinois at Urbana-Champaign in 1996. He has held visiting appointments at Princeton University, Rice University, and UCLA. His interests lie in the broad area of information theory and signal processing, with applications in wireless communications. He was the secretary for the IEEE Information Theory Society in 2010-2011 and was the treasurer for ISIT 2010 in Austin, Texas. He has served as editor for the IEEE TRANSACTIONS ON INFORMATION THEORY, IEEE TRANSACTIONS ON WIRELESS COMMUNICATIONS, IEEE SignAl PROCESSING LETTERS, IEEE TRANS ACTIONS ON Image Processing, and IEEE Wireless Communications Magazine. He has been the recipient of the National Science Foundation career award, and is a fellow of IEEE. 